\title{
Discovery of Young Methane Dwarfs in the Rho Ophiuchi L 1688 Dark Cloud
}

\author{
Poshih Chiang and W. P. Chen \\ Graduate Institute of Astronomy, National Central University, 300 Jhongda Road, Zhongli \\ 32001, Taiwan
}

Received — ; accepted 


\begin{abstract}
We report the discovery of two methane dwarfs in the dark cloud L 1688 of the $\rho$ Oph star-forming region. The two objects were among the $\mathrm{T}$ dwarf candidates with possible methane absorption and cool atmospheres, as diagnosed by infrared colors using deep WIRCam/CFHT HK plus $\mathrm{CH}_{4} \mathrm{ON}$ images, and IRAC/Spitzer c2d data. Follow-up spectroscopic observations with the FLAMINGOS-2/Gemini South confirmed the methane absorption at $1.6 \mu \mathrm{m}$. Compared with spectral templates of known $\mathrm{T}$ dwarfs in the field, i.e., of the old populations, Oph J162738-245240 (Oph-T3) is a T0/T1 type, whereas Oph J162645-241949 (Oph-T17) is consistent with a T3/T4 type in the $\mathrm{H}$ band but an L8/T1 in the K band. Compared with the BT-Settl model, both Oph-T3 and Oph-T17 are consistent with being cool, $\sim 1000 \mathrm{~K}$ and $\sim 900 \mathrm{~K}$, respectively, and of low surface gravity, $\log (g)=3.5$. With an age no more than a couple Myr, these two methane dwarfs thereby represent the youngest $\mathrm{T}$ dwarfs ever confirmed. A young late L dwarf, Oph J162651-242110, was found serendipitously in our spectroscopic observations.
\end{abstract}

Subject headings: brown dwarfs; stars: formation; infrared: stars; stars: individual (Oph-L, Oph-T3, Oph-T17) 


\section{Introduction}

Thousands of brown dwarfs have been discovered in the field by large surveys such as SDSS (Knapp et al. 2004; Chiu et al. 2006; Scholz et al. 2009), 2MASS/DENIS (Kirkpatrick et al. 2000; Tinney et al. 2005; Reid et al.|2008; Martín et al. 2010), UKIDSS (Burningham et al. 2013), and WISE (Kirkpatrick et al. 2011, 2012). Recent discoveries of Y dwarfs push the mass of brown dwarfs down to some 10 Jupiter masses (Kirkpatrick et al. 2012). Furthermore, a few isolated, or possible members of young moving groups (100200 Myr), planetary mass objects, which have masses lower than the deuterium-burning limit, were recently identified with different spectral types, ages, and environments (Delorme et al. 2012; Liu et al. 2013; Luhman 2014; Naud et al. 2014; Gagné et al. 2015).

The current paradigm is that stars are formed out of dense molecular cloud cores, and planets are condensed in the protostellar disks. The origin of substellar objects, however, remains unsettled.

To shed light on the issue, identification of substellar objects in a star-forming region, i.e., at an epoch when these objects are being formed, is a crucial first step. Most brown dwarfs found in nearby star-forming region so far are of late-M or L types, e.g. OTS 44 in Chamaeleon (Oasa et al. 1999; Luhman et al. 2004), 2M0437+2331 in Taurus (Bowler et al. 2014), and CFHTWIR-Oph33 in $\rho$ Oph (Alves de Oliveira et al. 2012). Toward later types, it has been a long battle to recognize the youngest $\mathrm{T}$ dwarfs to even the closest star-forming regions, though some candidates have been reported, e.g., in IC 348 (Burgess et al. 2009), and in Serpens (Spezzi et al. 2012), both of 1-3 Myr. Field brown dwarfs appear to show the transition from spectral type $\mathrm{L}$ (cloudy, red, and dusty) to T (cloudless, blue, and methane-bearing) around $~ 1400 \mathrm{~K}$ (Kirkpatrick 2005). In contrast, young exoplanets and free-floating planetary-mass objects are expected to have the spectral transition at lower temperatures, possibly as the result of different grain physics in cool 
atmospheres (Bowler et al. 2011; Barman et al. 2011; Liu et al. 2013). To date, three controversial cases of very young $\mathrm{T}$ dwarfs were claimed, respectively, with two in $\sigma$ Ori, S Ori 70 (Zapatero Osorio et al. 2002) and S Ori 73 (Bihain et al. 2009), and one in $\rho$ Oph, No. 4450 (Marsh et al. 2010). However, recent proper motion data indicate S Ori 73 likely to be a field T dwarf, while the nature of S Ori 70 remains elusive (Peña Ramírez et al. 2011). On the other hand, No. 4450 does not show prominent methane features. A sample of confirmed methane dwarfs of a few million years old, therefore, will provide constraints to the theoretical modeling of the evolving cool atmospheres. Here we report the confirmation by infrared spectroscopy of two T dwarfs in the L 1688 cloud of the Rho

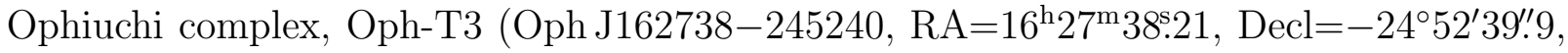
$\mathrm{J} 2000, H=18.38 \pm 0.06, H-K=0.28 \pm 0.09)$ and Oph-T17 (Oph J162645-241949, $\mathrm{RA}=16^{\mathrm{h}} 26^{\mathrm{m}} 45.23$, Decl $\left.=-24^{\circ} 19^{\prime} 49^{\prime} \cdot 2, \mathrm{~J} 2000, H=19.16 \pm 0.15, H-K=0.49 \pm 0.17\right)$, following the identification numbering in Table 3 of Chiang et al. (2015).

\section{Observations and Data Reduction}

\subsection{Photometric observations}

The T dwarfs candidates in L 1688, the densest cloud in Rho Ophiuchi, were identified by cool, methane-bearing atmospheres that characterize T dwarfs (Chiang et al. 2015). The methane absorption is diagnosed by our deep methane imaging survey carried out in 2010 using the $\mathrm{CH}_{4} \mathrm{ON}$ filter, centering at $1.69 \mu \mathrm{m}$, with the Wide-field InfraRed Camera (WIRCam) attached to the 3.6-m Canada-Frace-Hawaii Telescope (CFHT). Together with the archival WIRCam/CFHT H-band images, the color $H-C H 4 O N$ serves to trace possible methane absorption near $1.6 \mu \mathrm{m}$. In addition, the color [3.6] - [4.5], available through the c2d catalog (Evans et al. 2007), is used to detect another methane feature at $3.4 \mu \mathrm{m}($ Patten et al. 2006; Leggett et al. 2010; Mace et al. 2013). 
For cool temperatures, both $H-[4.5]$ and $K-[4.5]$ colors are utilized (Patten et al. 2006; Leggett et al. 2010). With these selection criteria, empirically adjusted with known M, L, and T dwarfs in the field, Chiang et al. (2015) found a total of $28 \mathrm{~T}$ dwarf candidates toward L 1688 that show evidence of both methane absorption and cool atmospheres. Notwithstanding about half of these 28 candidates may be contaminations, likely by active galaxies or young stellar variability (Chiang et al. 2015), the list provides a conservative, yet relatively reliable candidate sample of methane dwarfs for spectroscopic confirmation. Figure 1 shows the distribution of these candidates, including the validated $\mathrm{T}$ and $\mathrm{L}$ dwarfs presented here, in the diagnostic color-color and color-magnitude diagrams.

\subsection{Spectroscopic Observations}

Six T dwarf candidates were observed with FLAMINGOS-2 on the Gemini-South Telescope in 2014, using the HK grism with a 6-pixel wide $\left(1.08^{\prime \prime}\right)$ slit for low-resolution $(R \sim 1200)$ spectra. Four of the targets turned out to be false positives, including one galaxy with emission lines (source No. 1 in Chiang et al. 2015, R.A.=16:28:10.04, Decl.=-24:49:12.2 (J2000)), one background star (No. 2, R.A.=16:28:14.19, Decl.=-24:50:53.5, J2000), and two low-temperature objects (No. 7, R.A.=16:27:12.80, Decl.=-24:49:55.2, J2000, and No. 19, R.A.=16:28:40.09, Decl.=-24:00:17.1, J2000) showing possible water absorption but no obvious methane absorption. In this paper, we present the results of the two confirmed methane dwarfs among the 28 candidates listed by Chiang et al. (2015), and the serendipitous discovery of one L dwarf. It took six and seven dithering points, each with a detector integration time of $300 \mathrm{~s}$, in an "ABBA" pattern to complete the observations, rendering a total exposure time of 1,800 seconds for Oph-T3 and 2,100 seconds for Oph-T17. An argon lamp was used for wavelength calibration. The standard star HIP 82271 (B9 V) was observed to remove telluric absorptions. 

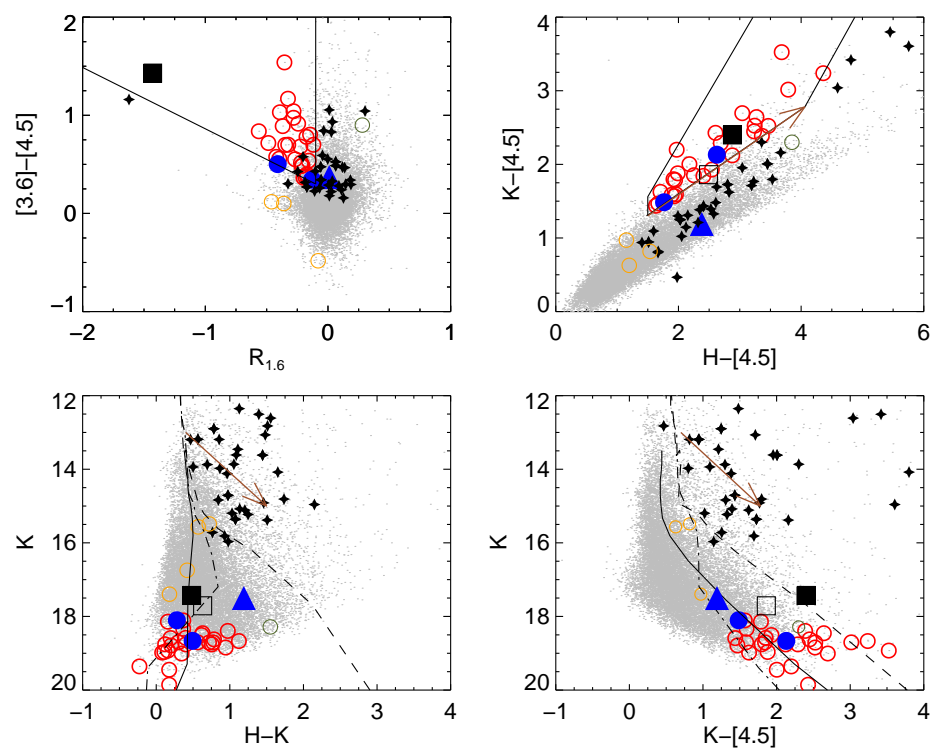

Fig. 1.- (Top) The color-color diagrams of (left) the methane indices, and (right) of the temperature indices. Gray dots represented all the sources in our data of L 1688 in Rho Ophiuchi. The region within which $\mathrm{T}$ dwarfs are identified (the "T zone") in each diagram is enclosed by solid lines. Stars located in both $\mathrm{T}$ zones are considered $\mathrm{T}$ dwarf candidates (red open circles), including the two confirmed $\mathrm{T}$ dwarfs (blue filled circles) and one $\mathrm{L}$ dwarf (blue filled triangle) reported here. Asterisks represent the spectroscopically confirmed late-M and L dwarfs in $\rho$ Oph (Geers et al. 2011; Alves de Oliveira et al. 2010; Mužić et al. 2012; Alves de Oliveira et al. 2012). Also marked are the suspected T dwarf candidates in other star-forming regions, such as S Ori 70 (Zapatero Osorio et al. 2002) (filled square) and No. 4450 (Marsh et al. 2010) (open square). No. 4450 has no detected methane feature, so it is not included in the methane diagram. The $\mathrm{T}$ dwarf candidates in IC 348 (green open circle, Burgess et al. 2009) and in Serpens (orange open circles, Spezzi et al. 2012) are also marked. Note that these would not have selected by our criteria. (Bottom) The colormagnitude diagrams, with the same symbols, overlaid with the 1 Myr isochrones by the COND (solid line) (Baraffe et al. 2003), DUSTY (dashed line) (Chabrier \& Baraffe 2000), and BT-settl (dot-dash line) (Allard et al. 2012) models. The magnitudes of S Ori 70 are scaled to 130 pc from its assumed distance 352 pc of the $\sigma$ Ori cluster. 
Raw data were processed with the standard F2/Gemini package under IRAF, including flat-field correction, cutting and co-adding images, wavelength calibration, telluric correction, and extraction of spectra. With the long exposures, sky emissions at 1.5, 1.58, 1.68 and $1.77 \mu \mathrm{m}$ were too bright to be properly removed so were discarded manually in subsequent analysis. Oph-T17 was observed before dawn with the airmass increasing from 1.7 to 2.7 , hence with an elevated thermal sky background. To minimize the effect of background changes, we scaled the images of each dither frame to the first frame. One additional bright star and one faint star, respectively, happened to be detected in the 2-D dispersed images of Oph-T3 and Oph-T17. Figure 2 shows the reduced and coadded 2D dispersed images, together with the extracted 1D spectra of Oph-T3 and Oph-T17. The bright star serves as a spectroscopic comparison for Oph-T3. The faint star coincidentally located in the slit of the Oph-T17 images, Oph J162651-242110, turned out to be a late L dwarf previously unrecognized.

\section{Spectral Typing}

We made two approaches for spectral classification of our candidates: (1) by comparison of the observed spectra with those of field brown dwarfs, and with atmospheric models, and (2) by spectral indices relevant to individual molecular absorption features.

Figure 3 shows the spectra of Oph-T3, Oph-T17 and Oph J162651-242110, all smoothed to facilitate visual comparison. Superimposed in the figure are the template spectra of field L and T dwarfs taken from the SpeX Prism Spectral Libraries (http://pono.ucsd.edu/ adam/browndwarfs/spexprism/publications.html), and the BT-Settl model spectra. In each case, the spectral running from the $\mathrm{H}$ to $\mathrm{K}$ band is normalized at $1.58 \mu \mathrm{m}$. 

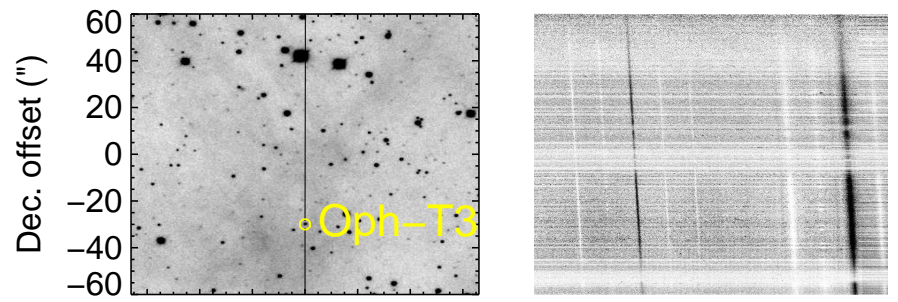

$604020 \quad 0-20-40-60$

R.A. offset (")

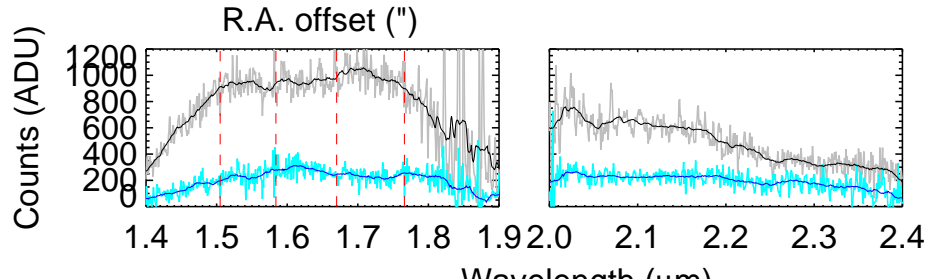

Wavelength $(\mu \mathrm{m})$
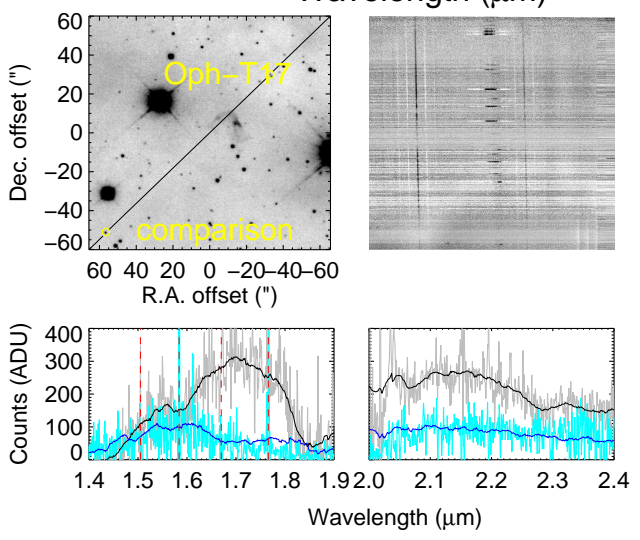

Fig. 2.- (Top) The observations of Oph-T3, showing (upper-left) the WIRCam $\mathrm{CH}_{4} \mathrm{ON}$ image, with the target Oph-T3 around $(0,30)$, a bright star around $(0,45)$, and the vertical solid line representing the position of the slit, (upper-right) the FLAMINGOS-2 2D dispersed image, with the thin and thick black stripes as Oph-T3 and the bright comparison star, (lower-left) the reduced H-band spectra for Oph-T3 (cyan) and the comparison (grey), each smoothed and represented by a thicker line, (lower-right) the reduced K-band spectra of the two stars. The red dashed lines in the $\mathrm{H}$ band mark the prominent sky emissions. (Bottom) The same as in the top but for Oph-T17. Oph-T17 is around $(-30,30)$ and its comparison around $(55,-50)$ in the $\mathrm{CH}_{4} \mathrm{ON}$ image. In the dispersed image, the spectra of Oph-T17, the emission nebula, and Oph J162651-242110 are seen from right to left. 
The template and model spectra have been all reddened using the empirical infrared reddening law prescribed by Indebetouw et al. (2005), namely $\log \left(A_{\lambda} / A_{K}\right)=$ $0.61-2.22 \log \lambda+1.21(\log \lambda)^{2}$. The amounts of extinction of our candidates, however, are highly uncertain. Chiang et al. (2015) estimated the extinction to each of their candidates on the basis of near-infrared star count. Oph-T17 $\left(A_{K} \sim 1.8\right)$ appears to suffer more extinction than Oph-T3 does $\left(A_{K} \sim=0.6\right)$. For Oph-T17, this must be an overestimate because the object is visible, albeit fainter, at shorter wavelengths. On the other hand, dereddening the observed color and magnitude along the interstellar reddening back to the model isochrone results in $A_{K} \sim 0.3 \mathrm{mag}$, but this estimate is susceptible to the uncertain validity of the adopted model isochrone, which is itself something we want to check. In Figure [3, we adopted a judicious value $A_{K} \sim 1.2$ mag for Oph-T17 to illustrate the effect of extinction on the emission continuum between $\mathrm{H}$ and $\mathrm{K}$ bands. The extinction has little influence on the methane line diagnosis covering a relatively narrow wavelength range. Likewise, $A_{K} \sim 0.8 \mathrm{mag}$ for Oph-T3 and $A_{K} \sim 0.3 \mathrm{mag}$ for the $\mathrm{L}$ dwarf Oph J162651-242110 are used in the plots, again for illustration, in each case by a consistent $\mathrm{H}$ to $\mathrm{K}$ band continuum and tracing roughly to the BT-Settl isochrone. A $\log (g)=3.5$ is used in the atmospheric models to compare to the observations.

Judging by the overall shape of the spectra, Oph-T3 should be a T0/T1 type, with the possibility of a late L. For Oph-T17, the spectrum in the $\mathrm{H}$ band is best represented by a T3/T4 type, but is more consistent in the $\mathrm{K}$ band with an earlier type, L8/T1. Note that the H-band spectrum resembles that of HR 8799e (Oppenheimer et al. 2013). The spectrum of Oph J162651-242110 in the H band exhibits possible water absorptions and follows reasonably well the L0 object 2M0437 in Taurus and the low-gravity exoplanet 2M1207b (Patience et al. 2010), and in the K band is consistent with BT-Settl models of $1100 \mathrm{~K}$ and low gravity, later than an L0. 
The discrepancy in spectral typing in the H- and K-band for Oph-T17 is not surprising, given the spectral templates being from the old, field populations. We compared the BT-Settl models of temperatures from 800 to $1100 \mathrm{~K}$ with various surface gravity values. The general trend is an enhanced suppression of the methane feature beyond $2 \mu \mathrm{m}$ with a lowering temperature, i.e., with a lower peak in the $2.10-2.15 \mu \mathrm{m}$ range, relative to that near $1.6 \mu \mathrm{m}$. Hotter than about $1000 \mathrm{~K}$, the methane absorptions disappear all together. A lower surface gravity tends to weaken the methane features. There is also the possibility of composite spectra due to binarity (Marocco et al. 2015). Both Oph-T3 and Oph-T17 are consistent with being cool, $\sim 1,000 \mathrm{~K}$, and $\sim 900 \mathrm{~K}$, respectively, and low surface gravity $(\log (g)=3.5$ bodies. The lack of an appreciable absorption longward to $\sim 2.3 \mu \mathrm{m}$ is perhaps due to an increased collision-induced $\mathrm{H}_{2}$ absorption with higher gravity (Burgasser, Burrow, \& Kirkpatrick 2006).

Alternatively, brown dwarf spectra can be classified by spectral index, i.e., the ratio of the flux across a molecular absorption (e.g., by water or methane) to that of the continuum, as demonstrated by the sets of spectral indices published by McLean et al. (2003); Allers \& Liu (2013) and Mace et al. (2013). Because our H band data are strongly affected by sky emissions, we modified the wavelength ranges of each spectral index as follows: $C H_{4}(A)=F_{1.730} / F_{1.570}=F(1.728,1.732) / F(1.593,1.597)$ (McLean et al. 2003), $\left(H_{2} \mathrm{O}-H\right)=F_{1.470} / F_{1.600}=F(1.450,1.490) / F(1.590,1.610)$, and $\left(C H_{4}-H\right)=F_{1.648} / F_{1.600}=F(1.635,1.660) / F(1.590,1.610)$ (Mace et al. 2013), where $F_{\lambda_{0}}=F(\lambda 1, \lambda 2)$ stands for the integrated flux from wavelengths $\lambda 1$ to $\lambda 2$, at central wavelength $\lambda_{0}$, all in microns. The $\mathrm{H}_{\text {con }}$ index is defined by the contrast between the "line" and two conninuum wavelengths (Allers \& Liu 2013). We adopted $\lambda_{\text {line }}=1.60$, the first continuum $\lambda_{1}=1.46$, the second continuum $\lambda_{2}=1.69$, and the modified $\mathrm{H}_{\text {con }}$ index becomes $\left[\left(\lambda_{\text {line }}-\lambda_{1}\right) F(1.68,1.70) /\left(\lambda_{2}-\lambda_{1}\right)+\left(\lambda_{2}-\lambda_{\text {line }}\right) F(1.45,1.47) /\left(\lambda_{2}-\lambda_{1}\right)\right] / F(1.59,1.61)$. 
The spectral types of Oph-T3 and Oph-T17 can be estimated by the modified spectral indices computed for known T dwarfs taken from the SpeX Libraries, as illustrated in Figure 4. Each spectral index is computed for a particular molecular feature with respect to the local continuum, so extinction has little effect. One sees that Oph-T3 is again consistent with a T0 type in all indices, whereas Oph-T17 shows a range of T0 to T4 types in different indices. In comparison to the BT-Settl models, a higher temperature corresponds to a higher gravity within $800-1600 \mathrm{~K}$. Both our targets are consistent with being cool and of $\operatorname{low} \log (g)$, and Oph-T17 is cooler than Oph-T3. For Oph J162651-242110, the $\left[\mathrm{H}_{2} \mathrm{O}\right]$ index suggests stronger water absorption than 2M0437+2331, a young L0 in Taurus, and the methane indices suggest no methane absorptions. We therefore conclude that Oph J162651-242110 is a young late L-type dwarf. The spectral types thus determined are listed in Table 1 .

Both $\mathrm{T}$ dwarfs reported here are too faint in our $\mathrm{J}$ images. Their (H-K) colors, $0.28 \mathrm{mag}$ for Oph-T3 and $0.49 \mathrm{mag}$ for Oph-T17, are comparable to intermediate-age T dwarfs or planet-mass objects, e.g., 0.30 mag for GU Psc b (50-120 Myr for the AB Doradus moving group, Naud et al. 2014), $0.54 \mathrm{mag}$ for CFBDSIR 2149-0403 (also for the AB Dor moving group, Delorme et al. 2012), and $0.60 \mathrm{mag}$ for 2MASS J01033563-5515561(AB)b (20-50 Myr for the Tucana-Horologium moving group, Delorme et al. 2013). Comparison of Oph-T3 and Oph-T17 does suggest a spectral sequence; in addition to the broad methane absorption beyond $1.6 \mu \mathrm{m}$, both objects share similar spectral characteristics, such as the absorption between 1.52 to $1.56 \mu \mathrm{m}$. Furthermore, Oph-T17 shows a more prominent methane absorption, and has a spectral running steeper than that of Oph-T3. We therefore conclude that OphT-17, being fainter, is cooler, i.e., of a later spectral type, than Oph-T3.

Our spectroscopic confirmation substantiates the photometric selection criteria presented by Chiang et al. (2015). In the two-color diagrams of Figure 1, Oph J162651-242110 
is located around the locus of young low-mass objects discovered in L 1688. In the color-magnitude diagrams, the three objects follow approximately the BT-settl isochrones in a spectral sequence. Including the L4 dwarf discovered by Alves de Oliveira et al. (2012) and the two $\mathrm{T}$ dwarfs reported here, the $\mathrm{L} / \mathrm{T}$ transition should happen between $\mathrm{K}=16$ to 18 mag, as the BT-settl model suggests.

In summary, two $\mathrm{T}$ dwarfs and one $\mathrm{L}$ dwarf in $\mathrm{L} 1688$ of the $\rho$ Oph star-forming region are identified on the basis of their $1.6 \mu \mathrm{m}$ methane absorptions and spectral morphology. Their spectral types are determined by comparing with the spectra and spectral indices of known L and T dwarfs in the field. Oph J162738-245240 (Oph-T3) is of a T0 spectral type. Oph J162645-241949 (Oph-T17) shows a T3/T4 type in the $\mathrm{H}$ band but an L8/T1 type in the $\mathrm{K}$ band. Both objects, when comparing with theoretical models, are consistent with having cool ( $900 \mathrm{~K})$ and low-gravity atmospheres. Oph J162651-242110 is a late L-type object. The three objects provide an anchor of the $\mathrm{L} / \mathrm{T}$ transition at $1 \mathrm{Myr}$ old. The discovery of the two $\mathrm{T}$ dwarfs validates our identification method using methane and temperature sensitive colors. Further high signal-to-noise ratio spectra observations obviously will determine the physical parameters much precisely.

We are grateful to Walfgang Brendner for help on data analysis, and to Catarina Alves de Oliveria for providing the spectrum of $2 \mathrm{M} 0437+2331$. We acknowledge the FLAMINGOS-2 team of Gemini South for the guidance on data reduction. Gratitude goes to the referee for constructive comments to substantially improve the quality of the paper. The project is financially supported by the MOST grant 103-2112-M-008-024-MY3.

Facilities: CFHT, Gemini South. 
Table 1. Properties of Brown Dwarfs in L 1688 in $\rho$ Oph

\begin{tabular}{ccccc}
\hline \hline Measurments & Unit & Oph-T3 & Oph-T17 & Oph J162651-242110 \\
\hline R.A. & hh:mm:ss.ss $(\mathrm{J} 2000)$ & $16: 27: 38$ & $16: 26: 45$ & $16: 26: 51$ \\
Dec. & dd:mm:ss.ss $(\mathrm{J} 2000)$ & $-24: 52: 40$ & $-24: 19: 49$ & $-24: 21: 10$ \\
$H$ & mag & 18.38 & 19.16 & 18.69 \\
$\mathrm{CH} 4 \mathrm{ON}$ & $\operatorname{mag}$ & 18.51 & 19.57 & 18.68 \\
$K_{s}$ & $\operatorname{mag}$ & 18.10 & 18.66 & 17.50 \\
{$[3.6]$} & $\operatorname{mag}$ & 16.96 & 17.04 & 16.68 \\
{$[4.5]$} & $\operatorname{mag}$ & 16.62 & 16.53 & 16.31 \\
$\left(\mathrm{H}_{2} \mathrm{O}-\mathrm{H}\right)$ & $F_{1.470} / F_{1.600}$ & $0.57(\mathrm{~T} 0)$ & $0.49(\mathrm{~T} 1)$ & 0.34 \\
$\left(\mathrm{CH}_{4}-\mathrm{H}\right)$ & $F_{1.648} / F_{1.600}$ & $0.95(\mathrm{~T} 0)$ & $0.70(\mathrm{~T} 3 / \mathrm{T} 4)$ & 1.28 \\
$\mathrm{H}_{\text {con }}$ & $(\mathrm{see} \mathrm{text)}$ & $0.89(\mathrm{~T} 0)$ & $0.60(\mathrm{~T} 3 / \mathrm{T} 4)$ & 1.08 \\
$\mathrm{H}_{2} \mathrm{O}(\mathrm{B})$ & $F_{1.456} / F_{1.570}$ & $0.49(\mathrm{~T} 0)$ & $0.54(\mathrm{~T} 0)$ & 0.23 \\
$\mathrm{CH}_{4}(\mathrm{~A})$ & $F_{1.730} / F_{1.595}$ & $0.86(\mathrm{~T} 0)$ & $0.36(\mathrm{~T} 4 / 5)$ & 1.44 \\
$\mathrm{CH}_{4}(\mathrm{~B})$ & $F_{2.200} / F_{2.100}$ & $0.98(\mathrm{~T} 0)$ & $0.91(\mathrm{~T} 0)$ & 0.93 \\
\hline
\end{tabular}

Note. - The wavelengths $(\mu \mathrm{m})$ of each modified spectral index is shown as the subscript in the second column. 


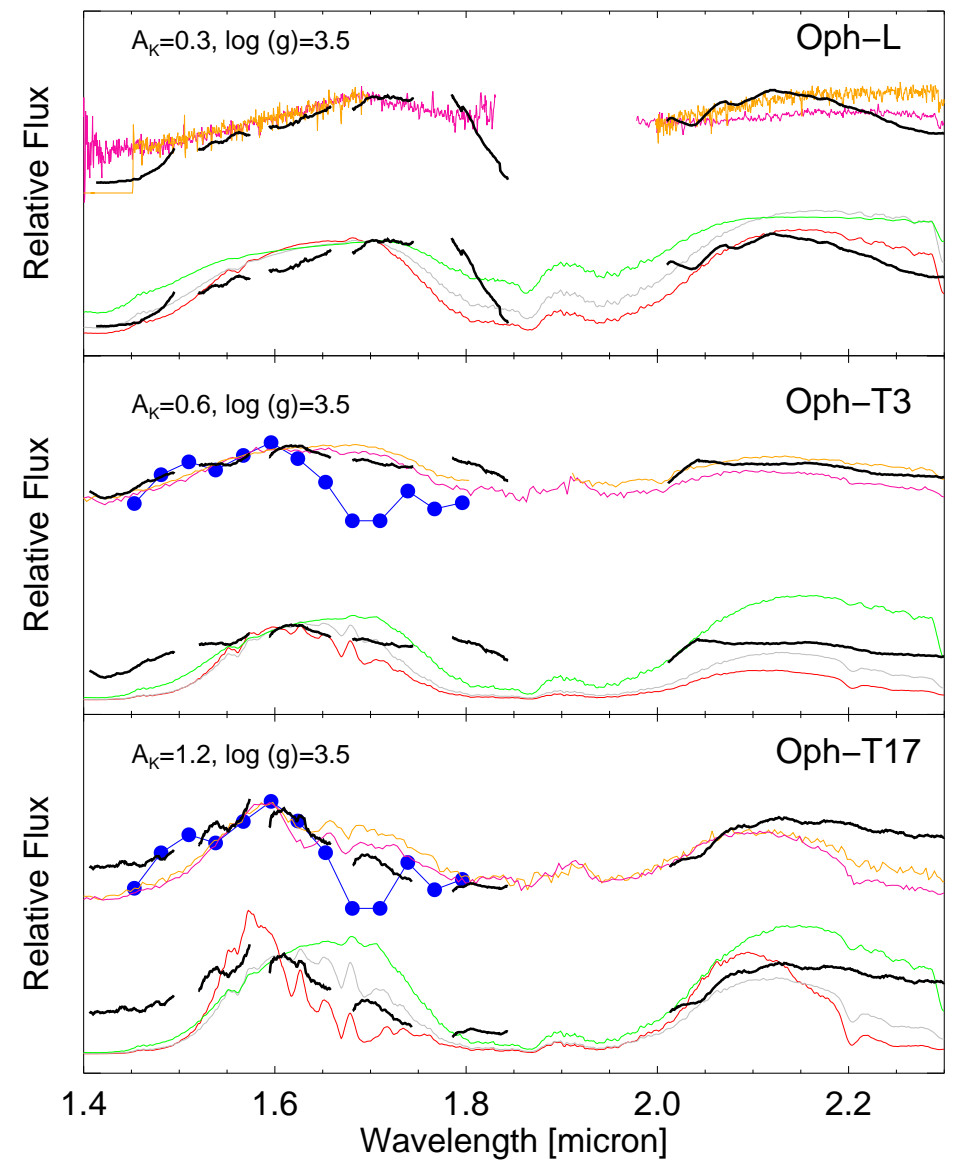

Fig. 3. - The spectra of brown dwarfs in L 1688 of the Rho Oph region. In every case, the top plot compares the spectrum of our target with other observations, whereas the bottom plot compares with BT-Settl models. (Top) The spectra of the young L dwarf (black), the L0 object 2M0437 (pink) in Taurus star-forming region (provided by Alves de Oliveria), the low-gravity exoplanet 2M1207b (orange) (Patience et al.|2010)), and the BT-Settl models of $1100 \mathrm{~K}$ (red), $1200 \mathrm{~K}$ (grey), and $1300 \mathrm{~K}$ (green). (Middle) The spectra of Oph-T3 (black), field L9 (orange) and T1 (pink) templates (red), and the BT-Settl models for $900 \mathrm{~K}$ (red), $1000 \mathrm{~K}$ (grey), and $1100 \mathrm{~K}$ (green), all for $\log g=3.5$. The blue dot-connected line in the $\mathrm{H}$ band shows the data of HR 8799e (Oppenheimer et al. 2013). (Bottom) The spectra of Oph-T17 (black), field T3 (orange) and T4 (pink) templates, and BT-Settl models for $800 \mathrm{~K}$ (red), $900 \mathrm{~K}$ (grey), and $1050 \mathrm{~K}$ (green), all for $\log g=3.5$. The blue dot-connected line in the $\mathrm{H}$ band again shows HR 8799e (Oppenheimer et al. 2013). 

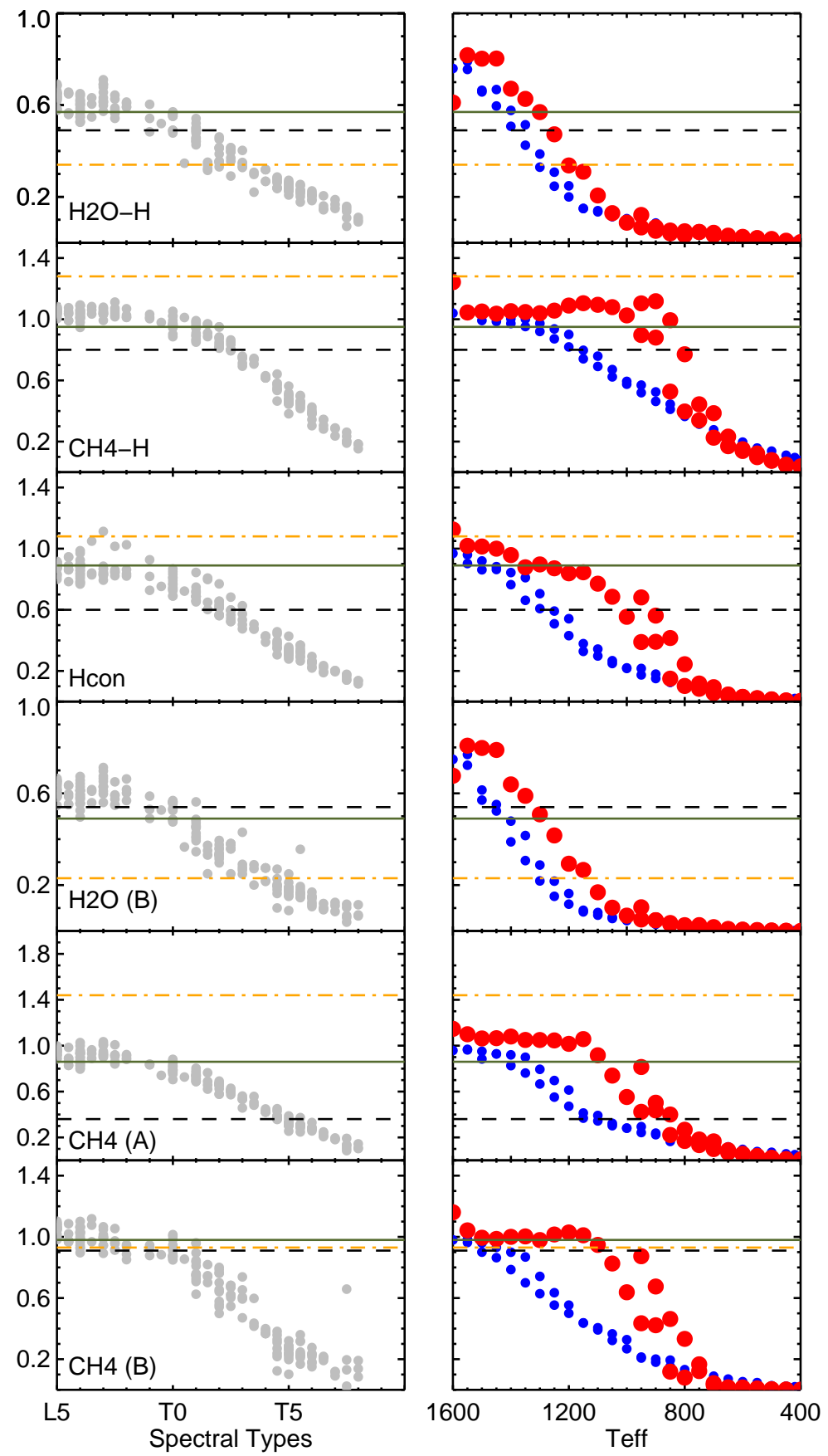

Fig. 4.- Spectral indices of (Left) field brown dwarfs (grey dots), and (Right) BT-Settl models with $\log (g) \geq 5.0$ (blue), and with $\log (g) \leq 3.5$ (red). Horizontal lines are for our Oph-T3 (solid green), Oph-T17 (dashed black), and the L object (dash-dotted orange). 


\section{REFERENCES}

Allard, F., Homeier, D., \& Freytag, B. 2012, Royal Society of London Philosophical Transactions Series A, 370, 2765

Allers, K. N., \& Liu, M. C. 2013, ApJ, 772, 79

Alves de Oliveira, C., Moraux, E., Bouvier, J., et al. 2010, A\&A, 515, A75

Alves de Oliveira, C., Moraux, E., Bouvier, J., \& Bouy, H. 2012, A\&A, 539, AA151

Barman, T. S., Macintosh, B., Konopacky, Q. M., \& Marois, C. 2011, ApJ, 735, LL39

Baraffe, I., Chabrier, G., Barman, T. S., Allard, F., \& Hauschildt, P. H. 2003, A\&A, 402, 701

Bihain, G., Rebolo, R., Zapatero Osorio, M. R., et al. 2009, A\&A, 506, 1169

Bowler, B. P., Liu, M. C., Kraus, A. L., Mann, A. W., \& Ireland, M. J. 2011, ApJ, 743, 148

Bowler, B. P., Liu, M. C., Kraus, A. L., \& Mann, A. W. 2014, ApJ, 784, 65

Burgasser, A. J., Burrows, A., \& Kirkpatrick, J. D. 2006, ApJ, 639, 1095

Burgess, A. S. M., Moraux, E., Bouvier, J., et al. 2009, A\&A, 508, 823

Burningham, B., Cardoso, C. V., Smith, L., et al. 2013, MNRAS, 433, 457

Chabrier, G., \& Baraffe, I. 2000, ARA\&A, 38, 337

Chiang, P., Chen, W. P., Albert, L., Liu, M., \& Magnier, E. A. 2015, MNRAS, 448, 522

Chiu, K., Fan, X., Leggett, S. K., et al. 2006, AJ, 131, 2722

Delorme, P., Gagné, J., Malo, L., et al. 2012, A\&A, 548, A26 
Delorme, P., Gagné, J., Girard, J. H., et al. 2013, A\&A, 553, L5

Evans N.J., Harvey P.M., Dumham M.M., Huard T.L., Mundy L.G., Lai S.P., Chapman N., Brooke T.Y., Enoch M.L., Stapelfeldt K.R., 2007, Final Delivery of Data from the c2d Legacy Project: IRAC and MIPS, (Pasadena: SSC)

Gagné, J., Burgasser, A. J., Faherty, J. K., et al. 2015, ApJ, 808, L20

Geers, V., Scholz, A., Jayawardhana, R., et al. 2011, ApJ, 726, 23

Indebetouw, R., Mathis, J. S., Babler, B. L., et al. 2005, ApJ, 619, 931

Kirkpatrick, J. D., Reid, I. N., Liebert, J., et al. 2000, AJ, 120, 447

Kirkpatrick, J. D. 2005, ARA\&A, 43, 195

Kirkpatrick, J. D., Cushing, M. C., Gelino, C. R., et al. 2011, ApJS, 197, 19

Kirkpatrick, J. D., Gelino, C. R., Cushing, M. C., et al. 2012, ApJ, 753, 156

Knapp, G. R., Leggett, S. K., Fan, X., et al. 2004, AJ, 127, 3553

Leggett, S. K., Burningham, B., Saumon, D., et al. 2010, ApJ, 710, 1627

Liu, M. C., Magnier, E. A., Deacon, N. R., et al. 2013, ApJ, 777, LL20

Luhman, K. L., Peterson, D. E., \& Megeath, S. T. 2004, ApJ, 617, 565

Luhman, K. L. 2014, ApJ, 786, LL18

Mace, G. N., Kirkpatrick, J. D., Cushing, M. C., et al. 2013, ApJS, 205, 6

Marocco, F., Jones, H. R. A., Day-Jones, A. C., et al. 2015, MNRAS, 449, 3651

Marsh, K. A., Kirkpatrick, J. D., \& Plavchan, P. 2010, ApJ, 709, L158 
Martín, E. L., Phan-Bao, N., Bessell, M., et al. 2010, A\&A, 517, AA53

McLean, I. S., McGovern, M. R., Burgasser, A. J., et al. 2003, ApJ, 596, 561

Mužić, K., Scholz, A., Geers, V., Jayawardhana, R., \& Tamura, M. 2012, ApJ, 744, 134

Naud, M.-E., Artigau, É., Malo, L., et al. 2014, ApJ, 787, 5

Oasa, Y., Tamura, M., \& Sugitani, K. 1999, ApJ, 526, 336

Oppenheimer, B. R., Baranec, C., Beichman, C., et al. 2013, ApJ, 768, 24

Patience, J., King, R. R., de Rosa, R. J., \& Marois, C. 2010, A\&A, 517, A76

Patten, B. M., Stauffer, J. R., Burrows, A., et al. 2006, ApJ, 651, 502

Peña Ramírez, K., Zapatero Osorio, M. R., Béjar, V. J. S., Rebolo, R., \& Bihain, G. 2011, A\&A, 532, AA42

Reid, I. N., Cruz, K. L., Kirkpatrick, J. D., et al. 2008, AJ, 136, 1290

Scholz, R.-D., Storm, J., Knapp, G. R., \& Zinnecker, H. 2009, A\&A, 494, 949

Spezzi, L., Alves de Oliveira, C., Moraux, E., et al. 2012, A\&A, 545, A105

Tinney, C. G., Burgasser, A. J., Kirkpatrick, J. D., \& McElwain, M. W. 2005, AJ, 130, 2326

Zapatero Osorio, M. R., Béjar, V. J. S., Martín, E. L., et al. 2002, ApJ, 578, 536 